\title{
Effects of sodium alendronate on osteoporosis and apoptosis-related factors Cyt C, Apaf-1 and caspase-9.
}

\author{
Hong Li, Chunde Li*, Xiaodong Yi, Hong Liu, Yu Wang \\ Department of Orthopedics, Peking University First Hospital, Beijing, PR China
}

\begin{abstract}
Objective: To investigate the clinical effect of alendronate on osteoporosis and its effect on Cyt C, Apaf-1 and caspase-9.

Methods: 100 cases of bone fracture patients diagnosed as osteoporosis in the hospital from January 2014 to December 2016. After fracture fixation, the patients were randomly divided into control group and observation group. The control group with 50 cases was given Calcium and observation group with 50 cases were treated with alendronate on the basis of the control group. The bone pain degree, bone mineral density and bone biochemical indexes of the two groups were recorded and compared. The cytochrome C (Cyt C) and Apoptotic Protease activating Factor-1 (Apaf -1) and caspase-9 levels of the two groups was compared.

Results: After treatment, the bone mineral density of both groups significantly increased, and the score of bone pain significantly decreased $(\mathbf{P}<\mathbf{0 . 0 5})$. After treatment, the bone mineral density of the observation group was significantly higher than that of the control group, and the visual score of bone pain was significantly lower than that of the control group $(\mathbf{P}<0.05)$. The level of serum osteocalcin in the observation group significantly increased and was significantly higher than that in the control group after treatment $(\mathbf{P}<\mathbf{0 . 0 5})$. The level of serum NTX in the observation group significantly decreased and was significantly lower than that in the control group after treatment $(\mathbf{P}<\mathbf{0 . 0 5})$. The serum calcium in both groups significantly increased after treatment $(P<0.05)$. The proportion of urinary calcium/ creatinine in the observation group was significantly lower than that in the control group after treatment. The levels of Cyt C, Apaf-1 and caspase-9 in the bone tissue of the observation group were significantly lower than those in the control group after treatment $(\mathbf{P}<0.05)$. In terms of safety, the incidence of adverse reactions was not significant differences $(\mathbf{P}>\mathbf{0 . 0 5})$.

Conclusion: Sodium alendronate can effectively ease the bone pain in osteoporosis patients, improve bone density, improve bone metabolism, and reduce the levels of apoptosis-related factors Cyt C, Apaf-1 and caspase- 9 in bone tissue of patients.
\end{abstract}

Keywords: Alendronate, Osteoporosis, Apoptosis-related factor, Efficacy.

Accepted on November 2, 2017

\section{Introduction}

Osteoporosis is bone mineral density and bone mass decline caused by various reasons. Because fine structure of bone is damaged, bone fragility increases, thus prone to the fracture of bone and other degenerative skeletal diseases [1]. With the development of economy and society in China, the trend of aging is becoming more and more obvious, and the incidence of osteoporosis is increasing year by year, which accounts for the fourth most common disease [2]. The skeleton of the human body is in constant metabolic process, the cause of osteoporosis is imbalance of bone metabolism and the balance between bone growth and resorption is broken [3]. Osteoblasts in bone tissue are related to bone formation, and osteoclasts are related to bone resorption. The two important causes of osteoporosis are reduced number and decreased activity of osteoblasts, as well as too high activity of osteoclastis [4]. The apoptosis related factors Cyt C, Apaf-1 and caspase-9 in bone cells can be used as a marker of apoptosis in bone tissue, which can indicate the damage degree of bone cells and severity degree of osteoporosis, but also can be used as a reference for the prognosis [5]. Allen phosphate is a bisphosphonate whose side chain contains nitrogen, which is widely used in the clinical treatment of osteoporosis patients. Its pharmacological effects are the inhibition of osteoclast function and the promotion of osteoclast apoptosis. However, studies have shown that Allen phosphate can promote the proliferation and transformation of osteoblasts [6]. A clinical study was carried out in this study on 100 cases of patients admitted to our hospital diagnosed as osteoporosis after fractures, aiming to explore and study the clinical effect of Allen phosphate in the treatment of patients with osteoporosis and its impact on 
apoptosis related factors Cyt C, Apaf-1 and caspase-9 in bone tissue cells, which are presented as follows.

\section{Data and Methods}

\section{General information}

Ethical approval was given by the medical ethics committee of Sixth People's Hospital of Qingdao with the following reference number: 2013022. In this study, patients diagnosed with osteoporosis after fracture were research subjects. All cases were 100 ones treated in our hospital from January 2014 to December 2016. After obtaining the approval from ethical committee and patient consent, the study patients were randomly divided by computer software into a control group and an observation group of respective 50 ones. The control group was given conventional calcium after the operation, including 21 males and 29 females; aged $62 \sim 78$ y old with an average of $(68.5 \pm 6.6 \mathrm{y}$ old $)$. The observation group was treated with Allen phosphate on the basis of the control group, including 22 males and 28 females; aged 63 77 (68.9 \pm 6.4$)$ years old. There was no significant difference between the two groups in the comparison of demographic data and course of disease, etc. $(\mathrm{P}>0.05)$.

\section{Inclusion and exclusion criteria}

Inclusion criteria: According to 'the guidelines for the diagnosis and treatment of osteoporotic fracture in China (the principle of the diagnosis and treatment for osteoporotic fracture)' and 'the guidelines for the diagnosis and treatment of primary osteoporosis, the patients were diagnosed as osteoporosis. Age $\geq 60$ y old using dual energy X-ray absorptiometry for measurement of lumbar Bone Mineral Density (BMD), T-score $\leq-2.5$ had no previous history of fracture before the occurrence of fracture. No history of longterm drugs use affecting the bone metabolism (glucocorticoids, sex hormones, etc.) and no anti-osteoporosis drugs use in half a year. No serious complicated diseases in patients' heart, lung, liver, kidney and blood system and no endocrine disease. The patients are informed and agree to donate bone tissue. Exclusive criteria: severe complicated diseases such as heart, brain, blood vessel, liver, kidney and blood system, tumor, or other diseases that can affect bone metabolism. Had endocrine diseases such as thyroid diseases, adrenal diseases, diabetes mellitus, etc. had congenital skeletal hereditary disease history of bone metabolism drugs use in 6 months. Patients had mental illness, Alzheimer's and so on, could not cope with the treatment of diseases. Patients did not agree to donate bone specimens.

\section{Treatment methods}

The control group: Received routine calcium for supplementation. D3 tablets of calcium carbonate (name of commodity: Caltrate D tablets, specifications: $600 \mathrm{mg} \times 30$, approval number: Otc H10950029, manufacturing enterprise:
Wyeth Pharmaceutical Co. Ltd) for oral use, 1 tablet per time, 2 times per day, taken in the morning and evening. Observation group: on the basis of the control group, they were treated with alendronate sodium, and the use method of calcium carbonate D3 tablets was similar to that of the control group, alendronate sodium (name of commodity: Fosamax, specifications: $70 \mathrm{mg}$ / tab, approval number: Otc J20130085, manufacturing enterprise: Merck Sharp \& Dohme Italia SPA (Italy) (Hangzhou moshadong Pharmaceutical Co. Ltd. Split packaging), taken $2 \mathrm{w}$ after surgical metal implantation for fixing fracture, once a week, one tablet per time, $70 \mathrm{mg}$ for oral use. The treatment courses of the two groups were for 1 year.

\section{Observation index}

Bone density index: DPX-1 dual energy X-ray absorptiometry bone density instrument was used to measure the bone density of lumbar 2 4 position and the femoral neck before treatment and $1 \mathrm{y}$ after treatment. The manufacturing company of the instrument was USA Lunar Company.

Score for bone pain: The "Changhai pain rule" was used as a standard for self-description of pain levels from the patients.

Bone metabolism index: Two groups of patients were extracted peripheral blood $5 \mathrm{ml}, 3000 \mathrm{rpm}$ centrifuge for 10 min, taken supernatant $0.5 \sim 1 \mathrm{ml}$ for preservation and further tested. Serum calcium, phosphorus, and creatinine were measured with a biochemical analyzer produced by instrument manufacturing company Beckman Company, USA. The urine at two time points was collected for measurement of calcium ion in urine. Use ELISA method to measure serum osteocalcin (BGP), Collagen $\mathrm{N}$ terminal peptide (NTX), of which the manufacturing company was BIORAD, USA and kit was purchased from Beijing Yinghua Company.

Apoptotic factor: Bone samples were taken from the fractured patients at the time of operation and after $1 \mathrm{y}$ when metal fixtures were taken and were cut into bone grain. They were washed by sterile physiological saline, frozen in liquid nitrogen and transferred to refrigerator at $-80^{\circ} \mathrm{C}$ for save. The bone tissue was polished by liquid nitrogen while detecting, and the protein was extracted as Western blot detection apoptotic factors Cyt, C, Apaf-1 and caspase-9.

Safety: The occurrence situation of adverse reaction in two groups was recorded, including gastrointestinal reactions, routine blood tests, and abnormal liver and kidney function, etc.

\section{Statistical methods}

SPSS18.0 was used in this study for statistical analysis of all data, mean \pm standard deviation was used to analyse the measurement data in two groups, count data were expressed by percentage, and Fisher's exact test as well as t-test were used. $\mathrm{P}<0.05$ was a significant difference in statistics. 


\section{Results}

\section{The degree comparison of bone mineral density index and bone pain between two groups of patients}

The bone mineral density of lumbar vertebral and femoral neck in patients in the two groups $1 \mathrm{y}$ after treatment had improved significantly $(\mathrm{P}<0.05)$, and the two indicators in observation group after one year of treatment were significantly higher than the control group $(\mathrm{P}<0.05)$. In 1 y after treatment, the bone pain score of patients in the two groups decreased significantly, and the score of bone pain in the observation group after $1 \mathrm{y}$ of treatment was significantly lower than that of the control group $(\mathrm{P}<0.05)$, as shown in Table 1 .

Table 1. Comparison of bone mineral density index between two groups of patients $(\bar{x} \pm s)$.

\begin{tabular}{|c|c|c|c|c|c|}
\hline Group & $\mathbf{n}$ & Time point & $\begin{array}{l}\text { Bone mineral density of lumbar } \\
\text { vertebral }\left(\mathrm{g} / \mathrm{cm}^{2}\right)\end{array}$ & $\begin{array}{l}\text { Bone mineral density of } \\
\text { femoral neck }\left(\mathrm{g} / \mathrm{cm}^{2}\right)\end{array}$ & Bone pain score \\
\hline \multirow[t]{2}{*}{ Control group } & 100 & Pretherapy & $0.67 \pm 0.08$ & $0.54 \pm 0.06$ & $6.35 \pm 1.45$ \\
\hline & & $1 \mathrm{y}$ after treatment & $0.71 \pm 0.08^{*}$ & $0.59 \pm 0.07^{*}$ & $3.44 \pm 1.34^{*}$ \\
\hline \multirow[t]{2}{*}{ Observation group } & 100 & Pretherapy & $0.67 \pm 0.07$ & $0.55 \pm 0.06$ & $6.33 \pm 1.32$ \\
\hline & & $1 \mathrm{y}$ after treatment & $0.76 \pm 0.08^{* \#}$ & $0.65 \pm 0.05^{* \#}$ & $1.67 \pm 1.55^{\star \#}$ \\
\hline
\end{tabular}

Note: "*" meant significant differences compared with that before treatment $(P<0.05)$; "\#" meant significant differences compared with that of the control group $(P<0.05)$.

\section{The comparison of bone metabolism indexes level of the two groups before and after treatment}

Serum calcium levels increased significantly in the two groups of patients after $1 \mathrm{y}$ of treatment $(\mathrm{P}<0.05)$. However, in $1 \mathrm{y}$ after treatment in the observation group, the serum osteocalcin level was significantly increased compared with that of pretherapy, and higher than that of the control group $(\mathrm{P}<0.05)$. Blood collagen $\mathrm{N}$ terminal peptide level decreased significantly compared with that of pretherapy, and lower than that of control group. The ratio of urinary calcium/creatinine decreased significantly compared with that before treatment, and higher than that of the control group $(\mathrm{P}<0.05$, Table 2$)$.

Table 2. Comparison of blood metabolism index levels between the two groups of patients before and after treatment $(\bar{x} \pm s)$.

\begin{tabular}{|c|c|c|c|c|c|c|c|}
\hline Group & $\mathbf{n}$ & Time point & $\begin{array}{l}\text { Blood calcium } \\
(\mathrm{mmol} / \mathrm{L})\end{array}$ & $\begin{array}{l}\text { Serum inorganic } \\
\text { phosphorus } \\
\text { (mmol/L) }\end{array}$ & $\begin{array}{l}\text { Serum osteocalcin } \\
(\mu \mathrm{g} / \mathrm{L})\end{array}$ & $\begin{array}{l}\text { Collagen } N \text { terminal } \\
\text { peptide }(\mu \mathrm{g} / \mathrm{L})\end{array}$ & Urinary calcium/creatinine \\
\hline \multirow[t]{2}{*}{ Control group } & 100 & Pretherapy & $1.94 \pm 0.12$ & $1.01 \pm 0.26$ & $12.13 \pm 1.21$ & $25.32 \pm 2.21$ & $0.20 \pm 0.04$ \\
\hline & & $\begin{array}{l}1 \quad y \quad \text { after } \\
\text { treatment }\end{array}$ & $2.36 \pm 0.21^{*}$ & $1.03 \pm 0.23$ & $11.91 \pm 1.24$ & $25.41 \pm 2.34$ & $0.19 \pm 0.05$ \\
\hline \multirow[t]{2}{*}{ Observation group } & 100 & Pretherapy & $1.96 \pm 0.14$ & $1.02 \pm 0.28$ & $12.16 \pm 1.25$ & $25.22 \pm 2.47$ & $0.19 \pm 0.06$ \\
\hline & & $\begin{array}{l}1 \quad y \quad \text { after } \\
\text { treatment }\end{array}$ & $2.39 \pm 0.25^{*}$ & $1.04 \pm 0.27$ & $14.62 \pm 1.27^{\star \#}$ & $20.33 \pm 1.67^{* \#}$ & $0.13 \pm 0.05^{\star \#}$ \\
\hline
\end{tabular}

Note: "*" meant significant differences compared with that before treatment $(\mathrm{P}<0.05)$; "\#" meant significant differences compared with that of the control group $(\mathrm{P}<0.05)$.

\section{Comparison of apoptosis related factors in bone tissue between two groups of patients}

There was no significant difference in bone tissue Cyt, C, Apaf-1 and Caspase-9 levels in the control group of patients before and after treatment $(\mathrm{P}>0.05)$. While in $1 \mathrm{y}$ after treatment, the levels of the three indexes in observation group decreased significantly, and significantly lower than those of the control group $(\mathrm{P}<0.05$, Table 3$)$.

Table 3. Comparison of islet function between the two groups of patients $(\bar{x} \pm s)$.

\begin{tabular}{llllll} 
Group & $\mathbf{n}$ & Time point & Cyt C & Apaf-1 & Caspase-9 \\
\hline $\begin{array}{l}\text { Control } \\
\text { group }\end{array}$ & 100 & Pretherapy & $0.67 \pm 0.21$ & $0.51 \pm 0.24$ & $0.79 \pm 0.34$ \\
\hline
\end{tabular}

\begin{tabular}{lccccc}
\cline { 2 - 5 } & & $\begin{array}{l}1 \quad \mathrm{y} \text { after } \\
\text { treatment }\end{array}$ & $0.66 \pm 0.31$ & $0.53 \pm 0.26$ & $0.76 \pm 0.32$ \\
\hline $\begin{array}{l}\text { Observatio } \\
\mathrm{n} \text { group }\end{array}$ & 100 & Pretherapy & $0.65 \pm 0.22$ & $0.52 \pm 0.27$ & $0.78 \pm 0.31$ \\
\cline { 2 - 5 } & & $\begin{array}{l}1 \mathrm{y} \text { after } \\
\text { treatment }\end{array}$ & $0.43 \pm 0.25^{* \#}$ & $0.42 \pm 0.28^{* \#}$ & $0.32 \pm 0.23^{* \#}$ \\
& & & & \\
\hline
\end{tabular}

Note: "*" meant significant differences compared with that before treatment $(P<0.05)$; "\#" meant significant differences compared with that of the control group $(\mathrm{P}<0.05)$.

\section{Comparison of safety}

During the treatment period in the two groups of patients, no cases of abnormal blood routine and liver and kidney function were found. In the observation group of patients, there were 3 cases of gastrointestinal reaction, and there was no significant 
difference in adverse reaction rate between the two groups (Fisher exact check $\mathrm{P}=0.243$ ).

\section{Discussion}

Osteoporosis is common in the elderly and postmenopausal women. It is a degenerative bone metabolic disease. With the aging process, nearly seventy million people over the age of 50 in China suffer from varying degrees of osteoporosis, while the incidence of total osteoporosis in people over the age of 60 is close to $40 \%$ [7-9]. Osteoporosis is often overlooked because of its concealed occurrence and unconspicuous symptoms. Many patients are diagnosed after fracture occurs. The bones of the human body are in constant metabolic process, and some studies suggest that $10 \%$ of the human skeleton has been renewed in 1 year. The cause of osteoporosis is imbalance between bone metabolism and bone remodeling, and the balance between bone growth and bone resorption are broken $[10,11]$. Bone resorption is carried out by osteoclasts, which receive cellular signal activation after formation, and release a series of inflammatory factors, cytokines, and digestive enzymes into bone tissue, leading to apoptosis of bone cells, destruction of skeleton microstructure, and playing the role of bone absorption [12,13]. Meanwhile, bone formation is carried out by osteoblasts. After osteoblasts receive the activation signal, they synthesize and release collagen and calcium binding proteins into bone tissue, and increase the formation and mineralization of bone microstructure. The two important causes of osteoporosis are the decreased number and activity of osteoblast as well as too high activity of osteoclast, resulting in the apoptosis of bone tissue and the destruction of bone microstructure [14,15]. The traditional drug treatment of osteoporosis is mainly calcium, but it has not been the first choice at present. This study investigated the clinical efficacy of alendronate sodium as a osteoclast inhibitor in the treatment of osteoporosis and its effects on bone metabolism and apoptosis related factors of bone tissue in the patients.

The bone mineral density of the lumbar vertebral body and the femoral neck increased significantly after $1 \mathrm{y}$ of treatment in the two groups of patients, while in the observation group, they were significantly higher than those in the control group. In the $1 \mathrm{y}$ after treatment, the bone pain score of the two groups of patients decreased significantly, and the score of observation group was significantly lower than that of the control group. It indicated that alendronate sodium could increase bone density and reduce bone pain more effectively. Serum calcium levels were significantly increased in the two groups of patients after $1 \mathrm{y}$ of treatment, which might be related to the use of calcium supplements in both of the two groups. While in $1 \mathrm{y}$ after treatment, the blood osteocalcin level of the observation group was significantly higher than that before treatment, and higher than that of the control group. The level of blood collagen $\mathrm{N}$ terminal peptide level was decreased significantly compared with that before treatment and significantly lower than that of the control group, and the ratio of urinary calcium/creatinine significantly decreased compared with that before treatment and significantly higher than that of the control group. Bone
Gla protein (BGP) reflects osteoblast activity, whereas the blood collagen $\mathrm{N}$ terminal peptide (NTX) reflects osteoclast activity, and urinary calcium/creatinine ratio reflects bone resorption levels. These results are similar to those of many studies [16,17], which shows that alendronate sodium can reduce osteoclast activity, decrease bone resorption, moreover, promote proliferation and differentiation of osteoblasts at low concentrations.

Cyt, C, Apaf-1 and Caspase-9 are apoptosis related factors released by mitochondria fragmentation in a series of cascade reactions after mitochondria receive apoptotic signals in the upstream pathway. It is a marker of apoptosis [5]. The levels of three indexes in bone tissue can reflect the extent of bone cell apoptosis and the trend of subsequent destruction of bone tissue. In this study, the control group was treated with calcium, and there was no significant difference between the three levels before and after treatment. While the control group was treated with alendronate sodium and after treatment, the three levels in bone tissue were significantly decreased, indicating that alendronate sodium might reduce the apoptosis of bone tissue cells by inhibiting osteoclast activity and other channels.

In conclusion, the results of this study suggest that alendronate sodium and calcium may be more effective in the treatment of osteoporosis, better in bone density increase and bone pain reduce, and more obvious in decreasing osteoclast activity, reducing bone resorption, as well as bone cell apoptosis. However, the sample size of this study is small, and more accurate results require larger samples and more evidence to confirm.

\section{References}

1. Liu $Z$, Xie $Z$. The role of common vitamin $D$ in the treatment of osteoporosis. J Clinic Int Med 2016; 33: 589-591.

2. Da W, Tang D, Shu B. Progress in the prevention and treatment of primary osteoporosis and vertebral compression fractures. Guangdong Med J 2016; 37: 1714-1717.

3. Chen X, Chen X. Eph/ephrin bidirectional signaling system and bone metabolism balance. Chinese J Biomed Eng 2016; 22.

4. Yan H, Guo J, An Y. Study progress in molecular signaling pathway in the pathogenesis of osteoporosis. Chinese J Osteopor 2016; 10: 1336-1340.

5. Zeng G. The correlation study between Bcl-2, Bak and osteoporosis as well as its TCM syndromes. Guangzhou Univ Trad Chinese Med 2016.

6. Yan X. Study on delivery system of hydroxyapatite loaded alendronate sodium drug with micron hollow structure. Jilin Univ 2015.

7. Yang L, Liu R. Application of Changhai pain rule assessment method in postoperative pain care in Department of orthopedics. Prim Med Forum 2015; 113-114. 
8. Qian W. Advances in the study of statins in the treatment of osteoporosis. Chinese J Bone Joint Inj 2015; 30: 334-335.

9. Luo L, Xue LI, Zeng LL. Study progress of statins in treatment of osteoporosis. J Reg Anat Oper Surg 2009; 274: 30557-30562.

10. Jiang P, Song K. The research progress of osteoclast and its differentiation regulation mechanism. Chinese J Bone Joint 2017; 6: 223-227.

11. Song CY, Peng B, Shen JY. Research on regulation mechanism of osteoclast differentiation. Zhongguo $\mathrm{Gu}$ Shang China j Orthop Traumatol 2015; 28: 580-584.

12. Meng Q, Zheng R, Zhu Y. Osteoclast differentiation factor and its signal transduction pathway. Int J Stomatol 2015; 42: $189-193$

13. Cong F, Liu J, Wang C. Ginsenoside Rb2 inhibits osteoclast differentiation through nuclear factor-kappaB and signal transducer and activator of transcription protein 3 signaling pathway. Biomed Pharmacother 2017; 92: 927.

14. Sagalovsky S. Osteoporosis and RANKL-RANK-OPG System 2015.
15. Yi T, Xi C, Ren Y. Study on regulation of wine-processed Dipsacus on OPG/RANK/RANKL axis system in osteoporosis rats. J Mod Med Health 2016.

16. Peng X, Lin B, Lingling YU. Effects of alendronate for treating primary osteoporosis on serum IGF-1 and IGFBP-3. Fujian Med J 2014.

17. Palacios S, Neyro J L, Ferrer J. Reduction of urinary levels of N-telopeptide correlates with treatment compliance in women with postmenopausal osteoporosis receiving alendronate. J N Am Menop Soc 2012; 19: 67-74.

\section{*Correspondence to}

Chunde Li

Department of Orthopedics

Peking University First Hospital

PR China 